\title{
440.
}

\section{NOTE SUR UNE TRANSFORMATION GÉOMÉTRIQUE.}

[From the Journal für die reine und angewandte Mathematik (Crelle), tom. LxviI. (1867), pp. 95, 96.]

LA lecture de la Note de M. Hesse, "Ein Uebertragungsprincip" (t. Lxvi. p. 15 de ce Journal) m’a suggéré les remarques suivantes:

Soient $\left(a_{1}, b_{1}, c_{1}, d_{1}\right),\left(a_{2}, b_{2}, c_{2}, d_{2}\right),\left(a_{3}, b_{3}, c_{3}, d_{3}\right)$ des constantes données, on peut supposer que les coordonnées $(x, y)$ d'un point quelconque dans un plan soient exprimées en fonctions des paramètres variables $(u, v)$ par les équations

$$
x=\frac{a_{1}+b_{1} u+c_{1} v+d_{1} u v}{a_{3}+b_{3} u+c_{3} v+d_{3} u v}, \quad y=\frac{a_{2}+b_{2} u+c_{2} v+d_{2} u v}{a_{3}+b_{3} u+c_{3} v+d_{3} u v} .
$$

En introduisant une nouvelle indéterminée $s$, ces équations peuvent être écrites dans la forme

$$
\begin{aligned}
& s x=a_{1}+b_{1} u+c_{1} v+d_{1} u v, \\
& s y=a_{2}+b_{2} u+c_{2} v+d_{2} u v, \\
& s=a_{3}+b_{3} u+c_{3} v+d_{3} u v ;
\end{aligned}
$$

pour des valeurs données des coordonnées $(x, y)$ la quantité $s$ est en général déterminée par une équation quadratique, et les paramètres $u$ et $v$ sont des fonctions linéaires données de $s$; il y a cependant deux cas particuliers qu'il convient de distinguer.

$1^{\circ}$. L'équation quadratique en $s$ peut avoir la racine $s=0$ et, débarrassée de ce facteur, se réduire par conséquent à une équation linéaire; ce cas particulier a lieu si

c. VII. 
la condition $(a b c)(b c d)=(a b d)(a c d)$ est remplie, où la notation $(a b c)$ désigne le déterminant

$$
\left|\begin{array}{lll}
a_{1}, & b_{1}, & c_{1} \\
a_{2}, & b_{2}, & c_{2} \\
a_{3}, & b_{3}, & c_{3}
\end{array}\right|
$$

Dans ce cas $u$ et $v$ sont des fonctions rationnelles de $(x, y)$ et la transformation a la signification géométrique suivante:

En considérant deux droites quelconques $L, M$ dans l'espace et en menant par le point donné $(x, y)$ la droite unique $G$ qui rencontre ces deux droites, on peut supposer que $u$ et $v$ soient des paramètres qui déterminent les positions des points de rencontre sur les deux droites respectivement; c. à. d. que $u$ soit la distance d'un point fixe sur la droite $L$ au point de rencontre avec la droite $G$, et de même que $v$ soit la distance d'un point fixe sur la droite $M$ au point de rencontre avec la droite $G$.

$2^{\circ}$. Supposons $b_{1}: c_{1}=b_{2}: c_{2}=b_{3}: c_{3}$, ou ce qui est au fond la même chose $b_{1}-c_{1}=0, b_{2}-c_{2}=0, b_{3}-c_{3}=0$; alors $s$ est déterminée par une équation simple, mais $u$ et $v$ ne sont plus des fonctions rationnelles de $s$; on voit que dans ce cas $u+v$ et $u v$ sont des fonctions rationnelles de $(x, y)$, et que par conséquent $u$ et $v$ sont les racines d'une équation quadratique qui contient $(x, y)$ linéairement. On peut supposer que $u$ et $v$ soient les paramètres de deux points sur une droite donnée, c. à. d. que $u$ et $v$ soient les distances de ces deux points respectivement à un point fixe situé sur la droite donnée; on a ainsi la transformation de M. Hesse.

Je n'ai pas cherché la signification géométrique des formules générales.

Cambridge, 10 octobre 1866. 